ISSN: 2386-3919 - e-ISSN: 2386-3927

DOI: https://doi.org/10.14201/et20173523754

\title{
ARMONIZACIÓN ENTRE LAS COMPETENCIAS DOCENTES Y DISCENTES EN EDUCACIÓN SECUNDARIA Y SUPERIOR
}

\section{Harmonization between teaching skills and students in secondary and higher education}

\author{
Reyna de los Ángeles CAmpa Álvarez*, Blanca Aurelia VAlENZuela** \\ y Manuela GUILLÉN LÚGIGO***** \\ Universidad de Sonora (México) \\ Correo-e: *reyna_angeles@botmail.com \\ **blancav@sociales.uson.mx \\ **mguillen@sociales.uson.mx
}

Recibido: 25-8-2016; Aceptado: 8-3-2017; Publicado: 30-11-2017

Ref. Bibl. REYNA DE LOS ÁNGELES CAMPA ÁLVAREZ, BLANCA AURELIA VALENZUELA y MANUELA GUILLÉN LÚGIGO. Armonización entre las competencias docentes y discentes en educación secundaria y superior. Enseñanza \& Teaching, $35,2-2017,37-54$.

RESUMEN: El presente estudio tiene como finalidad evaluar las competencias discentes de los estudiantes de la División de Ciencias Sociales de la Universidad de Sonora, México, y comprobar la armonización entre las competencias docentes del profesorado, a través de la formación previa recibida a nivel básico en educación secundaria y media superior. Se empleó una metodología cuantitativa de tipo descriptivo-correlacional, se aplicó el cuestionario-escala AMECESU «Formación del profesorado para la armonización de Modelos de Enseñanza-aprendizaje y desarrollo de Competencias en Educación Secundaria y Universitaria", a una muestra de 298 alumnos de la División de Ciencias Sociales de la Universidad de Sonora, México. Los datos obtenidos se analizaron con los programas estadísticos SPSS versión 21 y EQS 6.1 (Bentler). Se obtuvieron datos descriptivos, correlaciones y el modelo de ecuaciones estructurales. Los resultados indican que el modelo planteado en su conjunto predice 
REYNA DE LOS ÁNGELES CAMPA, BLANCA AURELIA VALENZUELA Y MANUELA GUILLÉN ARMONIZACIÓN ENTRE LAS COMPETENCIAS DOCENTES Y DISCENTES

EN EDUCACIÓN SECUNDARIA Y SUPERIOR

un 34\% de las competencias discentes a nivel de educación superior. Se puede concluir, por lo tanto, que la formación en competencias a nivel básico (secundaria) y medio superior (bachillerato) tiene una relación altamente significativa y se armoniza con la formación que actualmente reciben los estudiantes en educación superior; son los profesores de ambos niveles educativos un elemento clave en el proceso de enseñanza-aprendizaje para la transmisión y adquisición de las competencias discentes de los futuros profesionales en el campo de las ciencias sociales.

Palabras clave: competencias docentes; competencias discentes; educación básica; educación media superior y educación superior.

SUMMARY: This study aims to assess the learners skills of students of the Division of Social Sciences of the University of Sonora, Mexico and while harmonization between teaching skills; through previous training received in basic level education and upper secondary education, in order to determine a model with the core elements of the learners skills level of higher education. A quantitative methodology descriptive correlational was employed, the questionnaire-scale AMECESU «Teacher training for the harmonization of models of teaching and learning and skills development in secondary and university education", a sample of 298 students applied the Division of Social Sciences of the University of Sonora, Mexico. The data obtained were analyzed in the statistical program sPSS version 21 and EQS 6.1 (Bentler), where data reliability, descriptive, correlation and structural equation model were obtained. The results indicate that the proposed model predicts a whole 34\% of learners responsibilities at higher education. It can be concluded therefore that training in skills at a basic level (secondary) and upper secondary (high school) has a highly significant relationship and harmonizes with currently receiving training students in higher education; It is teachers of both levels of education a key element in the process of teaching and learning for the transmission and adquisition of skills of learners coming professionals in the field of social sciences.

Key words: teaching skills; learners skills; basic education; secondary education and higher education.

\section{INTRODUCCIÓN}

En los años noventa en México y en otros países surgió la tendencia mundial de reformar los planes de estudio bajo el enfoque de la educación basada en competencias (OCDE, 2002). Por lo cual, la modificación de los programas de formación que se implementan a partir de la definición de un conjunto de competencias genéricas y específicas que conforman los perfiles de egreso, como es el caso de las reformas desarrolladas en México en los diversos niveles educativos tanto básico, medio superior y superior (García, Loredo y Carranza, 2009).

La tendencia de implementar el enfoque por competencias consistió en cambiar los modelos educativos, los planes y los programas de estudio bajo el enfoque por competencias (Ocampo, 2003). La Universidad y su planta académica tienen un 
REYNA DE LOS ÁNGELES CAMPA, BLANCA AURELIA VALENZUELA Y MANUELA GUILLÉN ARMONIZACIÓN ENTRE LAS COMPETENCIAS DOCENTES Y DISCENTES

EN EDUCACIÓN SECUNDARIA Y SUPERIOR

gran reto ante sí: deben adaptarse a los cambios ocurridos en las últimas décadas en la sociedad para continuar con su misión más importante: la formación de los profesionales que la sociedad requiere y la construcción del conocimiento. Siendo así por lo que la demanda de profesionales con un buen nivel competencial avanza rápidamente (Informe Pisa, 2006).

El uso de la noción de competencias se presenta como alternativa a modelos formativos que han sido insuficientes para dar respuesta a las necesidades laborales y a los problemas que depara la vida (Zabalza, 2007: 31). Se puede definir competencia como "la capacidad o habilidad de efectuar tareas o hacer frente a situaciones diversas de forma eficaz en un contexto determinado, y para ello es necesario movilizar actitudes, habilidades y conocimientos al mismo tiempo y de forma interrelacionada» (Zabalza, 2007: 43 y 44).

En el mismo sentido, Moreno y Menjivar (2011) plantean que las competencias son capacidades complejas, que representan distintos grados de integración y se expresan en una gran variedad de situaciones en los diversos ámbitos de la vida humana personal y social. Indican que las competencias poseen tres dimensiones del estudio que son clave como lo es lo cognitivo, interpersonal y afectivo, donde una interrelación entre ellas por lo que se da un ejercicio de comunicación. Sin embargo, el informe Tuning en año 2005 reporta que el concepto de competencias convoca a una combinación de atributos en relación a los conocimientos, aptitudes, destrezas y responsabilidades que se manifiesta con la capacidad de desempeño en la persona.

Por su parte, Medina (2010) describe las competencias docentes como claves para facilitar el desarrollo de los alumnos, donde la formación en competencias es esencial y exige una tarea compleja e intensa, que implica a los saberes, destrezas, actuaciones y compromisos, que van caracterizando al ser humano a partir de las demandas del medio social y de los retos que implica la innovación de los perfiles profesionales que se requieren. Asimismo, consideran un requisito básico la formación del profesorado en las competencias genéricas, por lo que «aprender a desarrollar las competencias genéricas como docentes y vivirlas en plenitud es el gran desafío del profesorado universitario" (Medina 2010: 32). Para ello es necesario considerar la realidad del contexto institucional y social. En este sentido, Sevillano (2009) plantea que la educación a lo largo de la vida se basa en cuatro pilares: aprender a hacer, aprender a vivir juntos, aprender a ser y aprender a aprender.

La creciente importancia dada al enfoque de competencias en el aprender a aprender el profesorado se deriva de la mayor consideración de su trabajo como una profesión que posee un perfil profesional específico y distinto al de otros profesionales. Lo que significa entender la práctica de la enseñanza como una profesión y derivar de ahí las implicaciones para la formación (Schön, 1992). Es así que en la práctica didáctica se extiende como el proceso de enseñanza-aprendizaje en cualquier nivel educativo y país. Demandando en los profesores que cuenten con un perfil profesional que les permita responder de una manera adecuada a los retos y demandas de la actual sociedad del conocimiento y de la información (Serrano y Pontes, 2015). 

ARMONIZACIÓN ENTRE LAS COMPETENCIAS DOCENTES Y DISCENTES

EN EDUCACIÓN SECUNDARIA Y SUPERIOR

La docencia centrada en el aprendizaje conlleva reconocer la importancia del docente universitario como guía de aprendizaje, aspecto que tiene relación con los rasgos de calidad de los programas educativos como son la presentación de contenidos, la metodología, las nuevas tecnologías y la elaboración de guías (Zavalza, 2007). La calidad de la enseñanza tiene un impacto clave en la calidad de qué y cómo aprende el estudiante (Hernández, Martínez, Da Fonseca y Rubio, 2005: 50). De ahí que los sistemas educativos hoy en día centren su atención en el aprendizaje del estudiante con el fin de desarrollar competencias adecuadas al contexto profesional, promoviendo la profesionalización, la transferencia y la calidad. De tal forma que las competencias están relacionadas con las actividades profesionales complejas, porque la competencia supone un poner en acción destrezas, aptitudes, comportamientos y actitudes, siendo así que la competencia implica acción un saber actuar, movilizar, combinar, transferir, validando y demostrando una situación profesional compleja, por lo que la competencia se contextualiza con ciertos fines (Le Boterf, 2005).

\section{MARCO TEÓRICO}

\subsection{Formación del profesorado en el modelo de competencias}

En la sociedad actual y ante los cambios derivados de la globalización, la educación tiene un gran reto, tanto en el acceso a la educación como en su aprovechamiento. Tiene ante sí el desafío de realizar la función primordial de preparar y generar en los estudiantes las destrezas, habilidades y conocimientos suficientes para enfrentar los desafíos del mundo actual y, conjuntamente, debe modificar, ajustar y adaptar perfiles docentes, planes de estudios, infraestructura y su funcionamiento, con la finalidad de generar las condiciones más adecuadas y propicias que le permitan ofrecer la educación de calidad requerida y exigida hoy en día (Valenzuela, Huerta, Rodríguez, Campa y Hurtado, 2013).

Por lo que las instituciones de educación superior asumen un gran reto en la formación de los futuros profesionistas, la educación que reciben debe estar enmarcada dentro de un contexto de aprendizaje que de respuesta a las demandas de los sistemas productivos y a la sociedad. Ante las exigencias actuales, es necesario que el profesorado cuente con una formación propicia para construir su propio mapa de competencias profesionales con las que desarrollar su labor educadora, en colaboración constante con el colectivo de profesores con los que comparte la tarea docente, para progresar en equipo y con alta implicación institucional (Domínguez, Leví, Medina y Ramos, 2014).

Siendo los profesores uno de los actores educativos más relevantes en los centros educativos. Los cuales tienen la responsabilidad de desarrollar una serie de estrategias con sus alumnos en una interacción, la mayoría del tiempo grupal o en su caso individual, de manera que puedan alcanzar un conjunto de metas y tratando de siempre de responder a las necesidades que presente el alumnado 
REYNA DE LOS ÁNGELES CAMPA, BLANCA AURELIA VALENZUELA Y MANUELA GUILLÉN ARMONIZACIÓN ENTRE LAS COMPETENCIAS DOCENTES Y DISCENTES

EN EDUCACIÓN SECUNDARIA Y SUPERIOR

(Salazar, Tinajero y Valenzuela, 2013). Por su parte, Rosales (2013) hace alusión a que el desarrollo y la innovación del profesorado se vincula estrechamente con las características de la formación docente tanto inicial como permanente; para ello es necesario que el profesional docente reciba estímulos y cuente con capacitaciones constantes que hagan posible la tarea innovadora.

Mencionado lo anterior es indispensable que la formación docente contemple el proceso integral de formación inicial de maestros, la actualización de profesores en servicio y el conjunto de acciones encaminadas a dotar al maestro de una mejor capacidad para su desempeño profesional (Rivera, 2008). Los profesores requieren prepararse para asumir voluntariamente las necesarias adaptaciones al servicio de las particulares necesidades de cada alumno. Debe, por tanto, formarse a los profesores para la selección de objetivos, actividades y prácticas de evaluación (Bauer y Shea, 2003: 45 en Gento, 2006).

Medina, De la Herrán y Sánchez (2011) plantean que en la función pedagógica, los profesores deben ser preparados para asumir, también, su rol de líderes sociales en la atención a la diversidad. Así pues: "Los educadores y educadoras socialmente comprometidos han de asumir que su intervención no se agota en el cambio individual, sino que han de intentar cambiar las condiciones sociales que provocan las desigualdades, la marginación o la descompensación social» (Vega, 2004: 105 en Gento, 2006).

La formación práctica de los profesionales de la educación es un proceso complejo que implica y atañe a las instituciones responsables de la capacitación de los futuros docentes. La toma de conciencia de esta necesidad concierne de manera singular a los responsables de la educación, dado que cada nuevo profesional que se responsabiliza de la mejora integral de los seres humanos ha de prepararse para dar respuesta a la multitud de circunstancias, vivencias y formas de búsqueda de sentido en la sociedad del conocimiento.

\subsection{Formación de los alumnos en competencias discentes}

La formación de las competencias es una tarea compleja, debido a la armonización que conlleva en niveles superiores de tal modo que se sintetice el conocimiento y la acción, de una manera integral. Demanda reconocer el "para qué» de lo aprendido, su relación con las necesidades vitales, a la vez que la aplicación de lo conocido y aportando el verdadero valor. Respondiendo en qué nos mejora integralmente cuanto sabemos y/o ponemos en práctica para descubrir, de nuevo, si somos y hemos avanzado durante el periodo evaluado en la "secuencia de logro" de cada competencia (Medina, Domínguez y Ribeiro, 2011).

Las competencias orientan y fundamentan los procesos formativos y propician nuevas maneras para avanzar en la mejora del acto docente, consolidando un nuevo estilo de toma de decisiones que afecta al conjunto de los componentes curriculares, esencialmente a: 
REYNA DE LOS ÁNGELES CAMPA, BLANCA AURELIA VALENZUELA Y MANUELA GUILLÉN ARMONIZACIÓN ENTRE LAS COMPETENCIAS DOCENTES Y DISCENTES EN EDUCACIÓN SECUNDARIA Y SUPERIOR

- Adaptación de los contenidos.

- Integración y complementariedad de los saberes para alcanzar las competencias.

- Metodología didáctica: sistema metodológico y estrategias docentes y discentes.

- Tareas y actividades.

- Medios didácticos.

- Modelos y pruebas de evaluación que han de tomar un especial significado en esta etapa, dado que los estudiantes han de entender la génesis, la secuencia y las decisiones más pertinentes para avanzar en el dominio integrado de las competencias a desarrollar.

Los alumnos son el eje fundamental que dará sentido al proceso enseñanza-aprendizaje, por lo que representa un gran reto, en función del cual se decidirá qué es lo que el estudiante necesita aprender para adquirir una formación integral (García, 2006). Así, identificar la armonización de las competencias permitirá reconocer sus implicaciones en la formación integral del estudiante.

La formación de las competencias de los estudiantes requiere el empleo complementario de medios didácticos clásicos y digitales; por lo que consiste en una preparación que integra y da un sentido holístico a cuanto adquiría el estudiante, al lograr sintetizar en la competencia el conjunto de dimensiones para lograr la más pertinente capacitación que los universitarios requieren ante los cambiantes desafíos de las profesiones. Es necesario que se contemplen los saberes académicos sustanciales a las disciplinas, que integran el corpus científico y tecnológico que cada estudiante ha de comprender, avanzar y asumir críticamente (Medina, Domínguez y Sánchez, 2008).

El conocimiento adquirido a partir de dichas competencias discentes ha de estar encaminado a la acción y práctica reflexiva en la toma de decisiones más adecuada para el futuro profesional. El aprender a hacer se concreta en el estilo de actuación y de conducta que cada persona ha de llevar a cabo en las más diversas circunstancias y especialmente en los que requiere en la práctica profesional.

El desarrollo de las competencias dependerá de la intensidad de las tareas y de la pertinencia de las mismas. Se proponen tantas tareas cuantas se estimen necesarias para el dominio de las competencias trabajadas, conscientes del papel de las actividades en la formación y capacitación profesional de los estudiantes, centrando esta innovación en el sentido y complementariedad de los medios didácticos (Medina, Domínguez y Sánchez, 2008).

\subsection{Educación secundaria y educación superior}

En México se denomina educación secundaria al nivel educativo que atiende a los alumnos entre 12 y 15 años. Se considera como el puente entre la educación elemental y la preparatoria. Este nivel educativo se puede dividir en secundaria 
REYNA DE LOS ÁNGELES CAMPA, BLANCA AURELIA VALENZUELA Y MANUELA GUILLÉN ARMONIZACIÓN ENTRE LAS COMPETENCIAS DOCENTES Y DISCENTES

EN EDUCACIÓN SECUNDARIA Y SUPERIOR

general, técnico y para trabajadores. Tienen una organización que considera la existencia de docentes por asignaturas. Las primeras son las de mayor presencia en el país. En las secundarias técnicas, además de proporcionar formación humanística, científica y artística, se brinda una educación tecnológica básica, y el alumno egresado obtiene un diploma que lo avala.

La Secretaría de Educación Pública (SEP) (2015) indica que un docente de Educación Secundaria que se desempeña eficazmente requiere, para el ejercicio de la docencia, tener un conocimiento sólido acerca de los propósitos, enfoques y contenidos del nivel educativo establecidos en el plan y los programas de estudio, al igual que de los procesos de aprendizaje y de desarrollo de los alumnos; además, debe contar con habilidades que le permitan analizarlos críticamente y revisar las tareas de su práctica profesional como referentes para asegurar que todos los alumnos de la escuela aprendan.

El propósito esencial del plan de estudios de la secundaria es contribuir a elevar la calidad de la formación de los estudiantes que han terminado la educación primaria, mediante el fortalecimiento de los contenidos que respondan a las necesidades básicas de aprendizaje de la población joven del país y que sólo la escuela puede ofrecer. Estos contenidos integran los conocimientos, las habilidades y los valores que permiten a los estudiantes continuar su aprendizaje con alto grado de independencia, dentro o fuera de la escuela; facilitan su incorporación productiva y flexible al mundo del trabajo; coadyuvan a la solución de las demandas prácticas de la vida cotidiana, y estimulan la participación activa y reflexiva en las organizaciones sociales y en la vida política y cultural de la nación.

A nivel de educación superior ha experimentado, a partir de la década de los noventa, un marcado interés por la calidad educativa, al reconocer en este nivel educativo que consiste en la principal herramienta para responder a las exigencias y demandas educativas en un contexto influenciado por los desafíos del proceso de globalización (Garbanzo, 2007).

En México la educación superior es una de las principales fuentes para el desarrollo social, político y económico. Hoy se cuenta con un sistema de educación superior diversificado y con amplia presencia nacional. El aumento de la población que cursa este nivel educativo va en crecimiento. La educación superior constituye un medio de educación en los jóvenes y las características de las instituciones están íntimamente relacionadas con la calidad de la formación de sus estudiantes (Guerrero, 2003).

La función principal de la educación superior es la formación de las personas en los distintos campos de la ciencia, tecnología, docencia, investigación y también la extensión de los beneficios de la educación y la cultura al conjunto de la sociedad, con el propósito de impulsar el progreso integral de la nación. 


\section{OBJETIVOS DE LA INVESTIGACIÓN}

\subsection{General}

Evaluar las competencias discentes de los estudiantes de la División de Ciencias Sociales de la Universidad de Sonora y a la vez la armonización entre las competencias docentes del profesorado, a través de la formación previa recibida a nivel secundaria y medio superior, con la finalidad de determinar un modelo con los elementos centrales de las competencias discentes en nivel de educación superior.

\subsection{Específicos}

1. Conocer la formación en competencias a nivel de educación secundaria y medio superior que recibieron los estudiantes universitarios.

2. Conocer la formación que tienen los estudiantes universitarios en competencias a nivel de educación superior.

3. Evaluar las competencias docentes del profesorado a nivel de educación secundaria y a nivel de educación superior.

4. Identificar la relación entre las variables de formación en competencias en los diversos niveles educativos, las competencias docentes del profesorado y la relación que tiene en las competencias discentes a nivel de educación superior.

\section{MÉTODO}

\subsection{Enfoque de la investigación}

En la presente investigación se emplea el enfoque cuantitativo, se utiliza para la recolección y análisis de datos para contestar preguntas de investigación o probar hipótesis. Se emplean la medición numérica, el conteo y el uso estadístico para establecer con exactitud los patrones de comportamiento en la población (Hernández, Fernández y Baptista, 2010).

\subsection{Tipo de investigación}

La investigación es de tipo descriptivo-correlacional, debido a que describe las variables relacionadas con las competencias discentes y a la vez conocer la relación entre las variables de formación en competencias a nivel de educación secundaria y superior; y las competencias docentes.

Los estudios miden, evalúan o recolectan datos sobre diversos conceptos (variables), aspectos, dimensiones o componentes del fenómeno a investigar. En un estudio descriptivo se selecciona una serie de cuestiones y se mide o recolecta información sobre cada una. Los estudios correlaciónales miden la relación de 2 
REYNA DE LOS ÁNGELES CAMPA, BLANCA AURELIA VALENZUELA Y MANUELA GUILLÉN ARMONIZACIÓN ENTRE LAS COMPETENCIAS DOCENTES Y DISCENTES

EN EDUCACIÓN SECUNDARIA Y SUPERIOR

o más variables, la utilidad y propósito de los estudios correlaciónales son saber cómo se puede comportar un concepto o variable (Hernández, Fernández y Baptista, 2010).

\subsection{Participantes}

Se empleó una muestra no probabilística por conveniencia, debido a que se tomó un subgrupo de la población total de los alumnos de la División de Ciencias Sociales de la Universidad de Sonora y fue por conveniencia ya que se tomaron una serie de criterios para la selección de los participantes.

La muestra total se conformó por 298 alumnos y los criterios de selección de los participantes fueron los siguientes:

- Alumnos de la División de Ciencias Sociales de la Universidad de Sonora, unidad regional centro.

- Alumnos que cursaran entre quinto y noveno semestre de las licenciaturas.

Se empleó una fase de recogida de datos:

1) Fase cuantitativa: Durante el segundo semestre del 2015 se aplicó el Cuestionario AMECESU «Formación del profesorado para la armonización de Modelos de Enseñanza-aprendizaje y desarrollo de Competencias en Educación Secundaria y Universitaria». Elaborado por Medina y Ramos, 2011.

\subsection{Técnicas de recolección de datos}

Se empleó la técnica cuestionario-escala, la cual consiste en una serie de preguntas estructuradas, que especifican en forma previa el grupo de alternativas de respuesta y el formato de la respuesta; estas pueden ser opción múltiple, dicotómica o de escala.

Se utilizó el Cuestionario AMECESU «Formación del profesorado para la armonización de Modelos de Enseñanza-aprendizaje y desarrollo de Competencias en Educación Secundaria y Universitaria». Elaborado por Medina y Ramos, 2011 (ver anexo 1). En donde se evaluaron los siguientes aspectos:

1. Datos demográficos.

2. Bloque 1. Competencias Discentes.

3. Bloque 2A. Formación en competencias nivel secundaria.

4. Bloque 2B. Formación en competencias nivel universidad.

5. Bloque 3. Armonización entre los niveles educativos de la enseñanza secundaria y universitaria.

La escala de evaluación utilizada es de tipo likert, con opciones 1 «Completamente en Desacuerdo" y 6 "Completamente de Acuerdo"; a la vez se utiliza 1 «Nada 

ARMONIZACIÓN ENTRE LAS COMPETENCIAS DOCENTES Y DISCENTES EN EDUCACIÓN SECUNDARIA Y SUPERIOR

formado» y 6 "Completamente formado». La duración de la aplicación es aproximadamente de 20 minutos.

\subsection{Procedimiento y análisis de datos}

Para la aplicación de los cuestionarios, se acudió a las aulas de los alumnos con autorización previa de los profesores asignados, se acudió a los diversos Departamentos de la División de Ciencias Sociales de la Universidad de Sonora.

La aplicación duró aproximadamente 30 minutos por aula, se acudió en el horario y día asignado por los respectivos profesores. Se brindaron las instrucciones y se indicó que respondieran con la mayor veracidad y se garantizó el anonimato.

Una vez concluida la recolección de datos se procedió a su respectivo análisis. Para el análisis cuantitativo se empleó la base estadística de datos spss versión 21.00 y el programa EQS 6.1 (Bentler), en donde se realizó un análisis estadístico para la fiabilidad y validez de los resultados, descriptivo, correlacional y se obtuvó un modelo estructural.

\section{Resultados}

Para la validez del instrumento, se obtuvó un alfa de Cronbach de .84 en las escalas que integran el instrumento. De acuerdo a los resultados encontrados referentes a las competencias discentes desarrolladas por los alumnos, se obtuvieron medias altas en que reciben una formación integral, se cuenta con un programa curricular basado en competencias, se transmiten valores sociales y en menor medida se muestran medias bajas en los métodos de enseñanza-aprendizaje por parte de los profesores; de igual forma las tareas no favorecen la adquisición de competencias, hay una falta de integración de medios didácticos y no se cuenta con una evaluación que permita medir las competencias desarrolladas por los estudiantes. En la Tabla 1 se muestran los datos descriptivos de las competencias discentes.

Realizando un comparativo entre las principales competencias a nivel secundaria y superior, podemos observar en la Gráfica 1 los porcentajes detectando que existe una formación previa que permite potencializar las competencias; a la vez se puede señalar una dificultad en la competencia de innovación. 
REYNA DE LOS ÁNGELES CAMPA, BLANCA AURELIA VALENZUELA Y MANUELA GUILLÉN ARMONIZACIÓN ENTRE LAS COMPETENCIAS DOCENTES Y DISCENTES

EN EDUCACIÓN SECUNDARIA Y SUPERIOR

\section{TABLA 1}

Descriptivos de competencias discentes

\begin{tabular}{|l|c|c|c|c|}
\hline \multicolumn{1}{|c|}{ COMPETENCIAS DISCENTES } & MEDIA & MEDIANA & MODA & D. E. \\
\hline Adquisición de competencias. & 4.26 & 4.00 & 5 & 1.244 \\
\hline Dominio de las competencias. & 4.29 & 4.50 & 6 & 1.409 \\
\hline Formación Integral. & 5.19 & 6.00 & 6 & 1.318 \\
\hline Programa curricular basado en competencias. & 4.34 & 4.00 & 5 & 3.225 \\
\hline Método de enseñanza-aprendizaje. & 3.97 & 4.00 & 5 & 1.292 \\
\hline Tareas para la adquisición de las competencias. & 4.04 & 4.00 & 4 & 1.346 \\
\hline Medios didácticos. & 4.14 & 4.00 & 5 & 1.287 \\
\hline Evaluación de las competencias. & 4.13 & 4.00 & 5 & 1.378 \\
\hline Solución de problemas. & 4.15 & 4.00 & 4 & 1.361 \\
\hline Valores sociales. & 4.45 & 5.00 & 5 & 1.264 \\
\hline
\end{tabular}

GRÁFICA 1

Comparativo entre competencias a nivel secundaria y superior

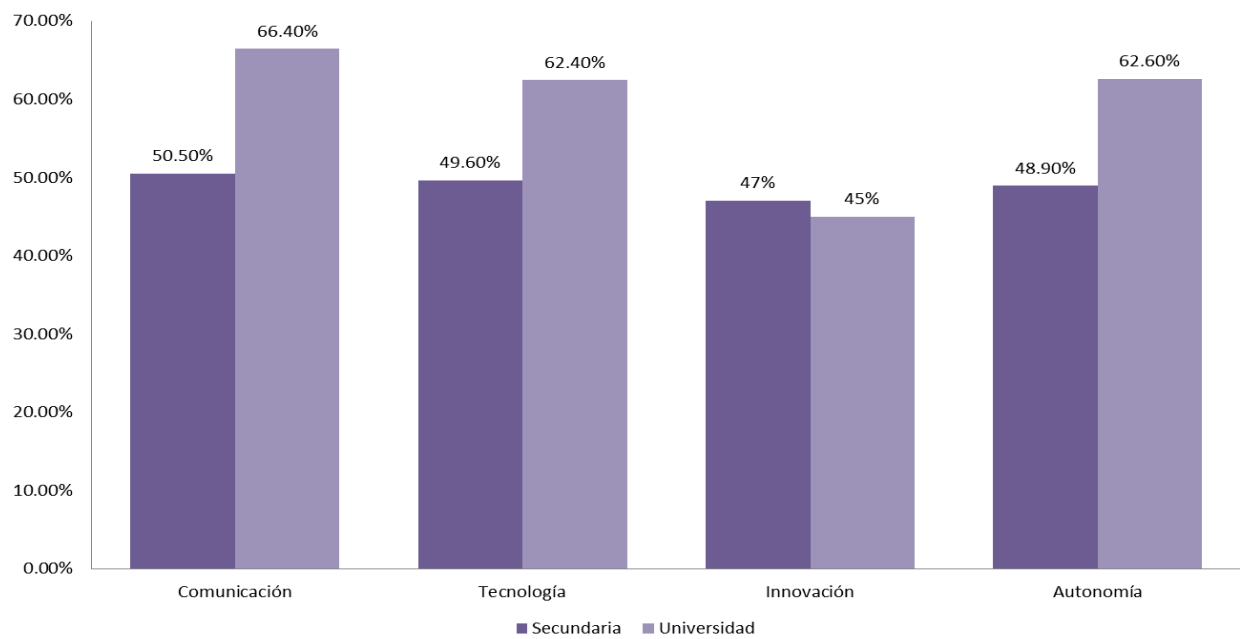

Evaluando las competencias de los docentes de secundaria y la formación previa al ingreso a la universidad, los alumnos evalúan que los profesores de universidad toman en cuenta la formación previa que recibieron los alumnos; sin embargo, los profesores de secundaria requieren emplear tareas adecuadas que los preparen para la Universidad. En la Tabla 2 se muestran dichos resultados. 

ARMONIZACIÓN ENTRE LAS COMPETENCIAS DOCENTES Y DISCENTES EN EDUCACIÓN SECUNDARIA Y SUPERIOR

TABLA 2

Armonización de competencias nivel secundaria y educación superior

\begin{tabular}{|l|c|c|c|c|}
\hline COMPETENCIAS & MEDIA & MEDIANA & MODA & D. E. \\
\hline $\begin{array}{l}\text { Los profesores de la universidad tie- } \\
\text { nen en cuenta los métodos, tareas y } \\
\text { prácticas que aplican los profesores } \\
\text { de secundaria. }\end{array}$ & 3.50 & 4.00 & 4 & 1.390 \\
\hline $\begin{array}{l}\text { Los profesores de secundaria emplean } \\
\text { métodos adecuados para preparar } \\
\text { para el trabajo en la universidad. }\end{array}$ & 3.44 & 4.00 & 4 & 1.439 \\
\hline $\begin{array}{l}\text { Los profesores de secundaria emplean } \\
\text { las tareas adecuadas para preparar } \\
\text { para el trabajo en la universidad. }\end{array}$ & 3.33 & 3.00 & 4 & 1.395 \\
\hline $\begin{array}{l}\text { Considera que los profesores de se- } \\
\text { cundaria emplean los medios adecua- } \\
\text { dos para preparar para el trabajo en la } \\
\text { universidad. }\end{array}$ & 3.38 & 3.00 & 4 & 1.363 \\
\hline $\begin{array}{l}\text { Considera que los profesores de se- } \\
\text { cundaria emplean las pruebas de eva- } \\
\text { luación adecuadas para preparar para } \\
\text { el trabajo en la universidad. }\end{array}$ & 3.41 & 3.00 & 4 & 1.361 \\
\hline
\end{tabular}

En la Tabla 3 se muestran las correlaciones de Pearson siendo significativos, principalmente, entre formación secundaria y formación educación superior (.711**), a la vez se relaciona la formación en educación superior y competencias discentes $\left(.566^{* *}\right)$ y entre competencias docentes y formación secundaria $\left(.440^{* * *}\right)$ y competencias discentes (.439**).

En la Figura 1, el modelo estructural, se obtuvo que la formación de competencias a nivel secundaria tiene una relación con las competencias discentes en la educación superior (coeficiente estructural $=.43$ ), es decir, que la formación que tienen como base los estudiantes en niveles anteriores impacta en las competencias que desarrollan durante la educación superior. Por otro lado, la formación que reciben a nivel universitario se relaciona altamente con las competencias discentes (.57); a la vez que las competencias docentes afectan en la adquisición de las competencias discentes en los estudiantes de educación superior (.44). Por último, se encontró que las competencias docentes son una variable mediadora en la formación de los estudiantes tanto en nivel secundaria como a nivel superior. Se puede encontrar validez convergente en los constructos que integran cada uno de los factores con pesos estructurales mayores de .5 , los cuales indican significancia. 
REYNA DE LOS ÁNGELES CAMPA, BLANCA AURELIA VALENZUELA Y MANUELA GUILLÉN ARMONIZACIÓN ENTRE LAS COMPETENCIAS DOCENTES Y DISCENTES

EN EDUCACIÓN SECUNDARIA Y SUPERIOR

TABLA 3

Correlaciones entre las variables de estudio

\begin{tabular}{|l|c|c|c|c|}
\hline & $\begin{array}{c}\text { FORMACIÓN } \\
\text { SECUNDARIA }\end{array}$ & $\begin{array}{c}\text { FORMACIÓN } \\
\text { EDUCACIÓN } \\
\text { SUPERIOR }\end{array}$ & $\begin{array}{c}\text { COMPETENCIAS } \\
\text { DISCENTES }\end{array}$ & $\begin{array}{c}\text { COMPETENCIAS } \\
\text { DOCENTES }\end{array}$ \\
\hline $\begin{array}{l}\text { Formación } \\
\text { secundaria }\end{array}$ & 1 & - & - & - \\
\hline $\begin{array}{l}\text { Formación } \\
\text { Educación Superior }\end{array}$ & $.711^{* *}$ & 1 & - & - \\
\hline $\begin{array}{l}\text { Competencias } \\
\text { Discentes }\end{array}$ & $.426^{* *}$ & $.566^{*}$ & 1 & 1 \\
\hline $\begin{array}{l}\text { Competencias } \\
\text { Docentes }\end{array}$ & $.440^{* * *}$ & $.369^{* * *}$ & $.439^{* * *}$ & - \\
\hline
\end{tabular}

* La correlación es significativa al nivel 0.01 (bilateral).

** La correlación es significante al nivel 0.05 (bilateral).

FIGURA 1

Modelo estructural de las VARIABles Del ESTUdio

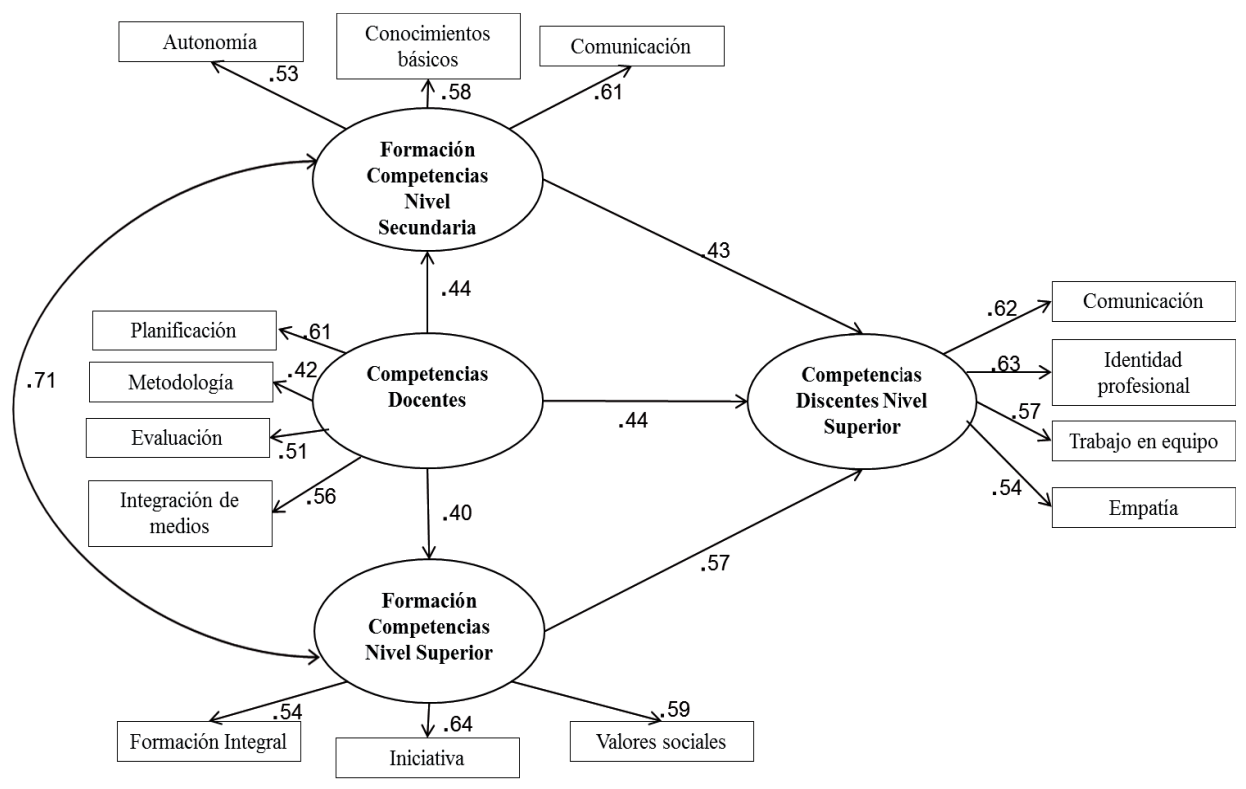



ARMONIZACIÓN ENTRE LAS COMPETENCIAS DOCENTES Y DISCENTES EN EDUCACIÓN SECUNDARIA Y SUPERIOR

Los indicadores de bondad de ajuste fueron considerados aceptables, ya que se obtuvieron los siguientes valores: índice de ajuste normado de Bentler-Bonett $($ BBNFI $)=.97$, índice de ajuste no normado de Bentler-Bonett $($ BBNNFI $)=.95 \mathrm{y}$ el índice comparativo de ajuste de Bentler $(\mathrm{CFI})=.96$. Estos valores alcanzan el nivel mínimo aceptable de los indicadores prácticos para considerar un modelo con buena bondad de ajuste que es de .90 . El valor del Chi cuadrado fue de X2 = 12.241 ( 8 g.1). $p=0.000$. La probabilidad no fue significativa, lo que indica que no existen diferencias entre el modelo teórico y el modelo saturado por las relaciones entre variables. Por último, se obtuvó un valor de .064 en RMSEA (Residuo de cuadrados mínimos), lo cual está dentro de los parámetros considerados aceptables .08. y la R2 fue .34, lo cual significa que el modelo en su conjunto explica el 34\% de la varianza competencias discentes en nivel de educación superior.

\section{CONClusiones}

De acuerdo a los resultados encontrados con el empleo de la metodología cuantitativa y los objetivos planteados en el estudio, permite brindar una explicación al objeto de estudio de competencias discentes y docentes. Se puede señalar primeramente, y como refieren autores como Medina, 2013; Domínguez, Medina y Chacheiro, 2010, y Zabalza, 2007, la formación en competencias a nivel de educación superior debe partir de un modelo basado en competencias, con un diseño de programas enfocados a la formación integral de los futuros profesionales.

Por lo que se encontró en la investigación planteada, la formación que reciben los alumnos de la División de Ciencias Sociales a nivel de educación superior indica que tienen una formación integral por parte de la Universidad de Sonora; también los planes y programas académicos se rigen bajo el enfoque basado en competencias. Un punto de gran relevancia es que las competencias discentes desarrolladas por los estudiantes son identidad profesional, trabajo en equipo, valores sociales y empatía en relación con su trabajo profesional.

Siguiendo los lineamientos por la Asociación Nacional de Universidades (ANUIES, 2015), se señala la relevancia que tiene la calidad de la educación superior, entendida como la eficiencia en los procesos, la eficacia en los resultados y la congruencia y relevancia de estos procesos y resultados con las expectativas y demandas sociales, es decir, el impacto y el valor de sus contribuciones con respecto a las necesidades y problemas de la sociedad. Siendo un elemento crucial para el éxito de las instituciones de la educación superior y de los cuales los profesores deben tener en cuenta en su ejercicio profesional.

En cuanto a las competencias docentes, los alumnos valoran que los profesores requieren una atención y preparación en cuanto a los métodos de enseñanza-aprendizaje, es necesario que el profesorado forme al alumnado con tareas encaminadas a la adquisición de competencias. A la vez se detecta una baja frecuencia en la integración de los medios didácticos en la práctica educativa. Por lo 
EN EDUCACIÓN SECUNDARIA Y SUPERIOR

último, no se cuenta con lineamientos claros y oportunos para la evaluación de las competencias adquiridas por los estudiantes.

Siendo, actualmente, la educación y la formación de los estudiantes un reto ante una sociedad cada vez más diversa y con desafíos para toda la comunidad educativa, especialmente en los profesores, quienes se encuentran frente al aula en constante interacción con los alumnos en su práctica docente demandando en este sentido el desarrollo de competencias docentes en el proceso de enseñanza-aprendizaje (Campa, Valenzuela, Guillén, Jaime y Enríquez, 2015).

Es necesario, por lo tanto, evaluar la formación recibida durante la educación básica debido a que, como indica Sarlé (2010), constituye un hito en la vida de los sujetos, representa la transición del niño y joven al territorio público y constituye una experiencia única e irrepetible de encuentro con otros adultos y niños(as). La asistencia y la inclusión de los niños y niñas al nivel básico los hará poseedores de múltiples conocimientos y experiencias propias y donde la relación pedagógica que se establezca será de vital importancia para la construcción de nuevos aprendizajes escolares y de vida.

En el estudio se encontró que la formación en competencias previa recibida por los alumnos en niveles de educación básica (secundaria) y educación media superior (bachillerato) fue principalmente en las competencias de comunicación, conocimiento del medio y autonomía que son en las que más se les formó a los estudiantes universitarios. Armonizando las competencias previas y en las competencias que actualmente se están formando a los estudiantes universitarios, se encuentra una relación con las competencias de comunicación, tecnología y autonomía. En ambos niveles educativos se encontró la necesidad de formar y reforzar la competencia discente de innovación, siendo la misma un elemento vital en el desarrollo de la práctica profesional.

Tal como lo plantea la Secretaría de Educación Pública, plantea tomar acciones para establecer los perfiles de desempeño de los docentes en servicio, con el fin de contar con una formación continua hacia el desarrollo de las competencias profesionales necesarias para afrontar los retos de la educación del siglo Xxi. Ante esto es necesario diseñar programas de capacitación en formación en competencias docentes enfocados en la atención adecuada de las innovaciones curriculares, gestión y especialmente del uso educativo de las tecnologías de la información y la comunicación.

La innovación de los perfiles docentes se ha de encaminar al impulso y la transformación que se requieren en los sistemas educativos para el aseguramiento de la calidad, por lo que necesitan estrategias institucionales dirigidas al fortalecimiento de los profesores. Las mismas instituciones educativas tienen como recurso primordial la formación y actualización competencial del profesorado como un capital de calidad.

Por último, el modelo planteado en general predice un 34\% las competencias discentes a nivel de educación superior. Se puede concluir, por lo tanto, que la formación en competencias a nivel básico (secundaria) y medio superior 

ARMONIZACIÓN ENTRE LAS COMPETENCIAS DOCENTES Y DISCENTES EN EDUCACIÓN SECUNDARIA Y SUPERIOR

(bachillerato) tiene una relación altamente significativa y se armoniza con la formación que actualmente reciben los estudiantes en educación superior; siendo los profesores de ambos niveles educativos un elemento clave en el proceso de enseñanza-aprendizaje para la transmisión y adquisición de las competencias discentes de los próximos profesionistas en el campo de las ciencias sociales. Cumpliendo de acuerdo a los resultados encontrados los objetivos de la investigación.

A manera de conclusión, la evaluación ha de formar parte del currículum universitario y del proyecto formativo, así como del sistema evaluativo de nivel medio. Por ello, el desarrollo de las competencias en los programas educativos ha de concordar y armonizar las competencias básicas requeridas a nivel secundaria y universitario. Donde el estudiante se convierte en el centro de innovación competencial, al que hay que favorecer en el proceso de enseñanza-aprendizaje; por ello, las competencias básicas se organizan y establecen a partir de la preparación de los docentes, del dominio de los contenidos a impartirse, de la selección de los métodos de enseñanza, de la comprensión de los objetivos que han de alcanzar los discentes (Valenzuela, Huerta, Rodríguez, Campa y Hurtado, 2013). En los niveles educativos evaluados, hay una exigencia en evaluar las competencias adquiridas por los alumnos y futuros profesionistas, para ello es importante hacer cambios importantes en las metodologías y herramientas de evaluación; a la vez que en el profesorado se debe evaluar su desempeño competencial (Tonda y Medina, 2013).

Algunas propuestas para futuras investigaciones serían ampliar el estudio utilizando una metodología mixta que permita triangular los datos y poder explicar el objeto de estudio; a la vez, involucrar en la muestra al profesorado y a los directivos correspondientes, de tal modo poder tener una información más amplia sobre la formación en competencias docentes y discentes. Debido a la importancia que tiene el conocer sus opiniones y sugerencias para la mejora de la educación. Por último, elaborar programas de intervención para el mejoramiento para la innovación en el proceso de enseñanza-aprendizaje.

\section{REFERENCIAS BIBLIOGRÁFICAS}

Asociación Nacional de Universidades e Instituciones de Educación Superior (ANUIES), (2015). Instituciones de Educación Superior. Descargado en febrero de 2016. http:// www.anuies.mx/anuies/instituciones-de-educacion-superior/pdf.

Bauer, A. M. P. y Shea, T. M. (2003) en Gento, S. (2006). Propuesta para una acción educativa de calidad en el tratamiento educativo de la diversidad. Revista Complutense de Educación, 17 (2), 13-34. ISSN: 1130-2496. Descargado en octubre de 2012. revistas. ucm.es/index.php/RCED/article/download/.../15786.

Campa, R.; Valenzuela, B.; Guillén, M.; Jaime, M. y Enríquez, J. (2015). Capítulo vi. Tratamiento educativo y práctica docente. Desafío para la calidad de la educación en atención a la diversidad. En B. Valenzuela, M. Guillén y A. Medina. Procesos educativos: desafios y retos en el siglo XXI. Pearson: México.

Domínguez, C.; Leví, G.; Medina, A. y Ramos, E. (2014). Las competencias docentes: diagnóstico y actividades innovadoras para su desarrollo en un modelo de educación a 
REYNA DE LOS ÁNGELES CAMPA, BLANCA AURELIA VALENZUELA Y MANUELA GUILLÉN ARMONIZACIÓN ENTRE LAS COMPETENCIAS DOCENTES Y DISCENTES EN EDUCACIÓN SECUNDARIA Y SUPERIOR

distancia. Revista de Docencia Universitaria, 12 (1), abril, 239-267. ISSN: 1887-4592. Recuperado en http://redu.net/redu/index.php/REDU/article/view/530.

Domínguez, G.; Medina, R. y Chacheiro, G. (2010). Investigación e Innovación de la Docencia Universitaria en Espacio de Educación Superior. España: Editorial Universitaria.

Garbanzo, G. (2007). Factores asociados al rendimiento académico en estudiantes universitarios. Una reflexión desde la calidad de la educación superior pública. Revista Educación, Universidad de Costa Rica, 31 (001), 43-63.

García, R. (2006). Las competencias de los alumnos universitarios. Revista Interuniversitaria de Formación de Profesorado, 20 (3), 253-269. España.

García, B.; Loredo, J. y Carranza, G. (2009). Análisis de la práctica educativa de los docentes: pensamiento, interacción y reflexión. Revista Electrónica de Investigación Educativa. Descargado en septiembre de 2012. http://redie.uabc.mx/NumEsp1/contenido-garcialoredocarranza.html.

Guerrero, J. (2003). Calidad en la educación, organizaciones y ejercicio profesional. Revista Alternativas en Psicología, 8, 24-35.

Hernández, R.; Fernández, C. y Baptista, P. (2010). Metodología de la investigación. Quinta edición. Perú: Editorial McGraw-Hill.

Informe Pisa (2006). Competencias científicas para el mundo de mañana. España: OCDE. Ministerio de Educación y Ciencia. Editorial Santilla.

Le Boterf, G. (2005). Ingeniería de las competencias. Barcelona: Epise Training Club y Ediciones Centros 2000.

Medina, A. (2010). Modelos de educación de la calidad en instituciones universitarias. Madrid: Edit. Universitad S.A.

Medina, A.; De la Herrán, A. y Sánchez, C. (2011). Formación Pedagógica y Práctica del Profesorado. España: Editorial Universitaria Ramón Areces, S.A.

Medina, A.; Domínguez, C. y Sánchez, C. (2008). Formación de las Competencias de los Discentes mediante un Diseño Integrado de Medios. EccoS Revista Científica, 10 (2), julio-diciembre, 327-357. Recuperado en http://www.redalyc.org/pdf/715/71511645004.pdf.

Medina, A.; Domínguez, M. y Ribeiro, F. (2011). Formación del profesorado universitario en las competencias docentes. Rev. hist. edu. latinoam., 13 (17), julio-diciembre. ISSN: 0122-7238, 119-138.

Medina, A.; Domínguez, C. y Sánchez, C. (2013). Evaluación de las competencias de los estudiantes: modelos y técnicas para la valoración. Revista de Investigación Educativa, 31 (1), enero, 239-255. Recuperado en http://www.redalyc.org/pdf/2833/283326290014.pdf.

Moreno, E. y Menjivar, S. (2011). Una mirada Internacional a las competencias docentes universitarias. España: Editorial Octaedro. ICE-UB.

Ocampo, F. (2003). El constructivismo en decadencia: de cómo fracasa su práctica pedagógica. Educere, 021, 119-124.

Organización para la Cooperación y el Desarrollo Económico (OCDE) (2002). Conocimientos y aptitudes para la vida. Resultados de PISA 2000. México: Santillana-OCDE.

Rivera, R. (2008). La Práctica Docente y la Formación Recibida en la Evaluación de la Calidad Educativa de Educación Superior. Tesis de Maestría. Centro Pedagógico del Estado de Sonora, Escuela Normal Superior de Hermosillo. Universidad Pedagógica Nacional. Recuperado en http://uva.ifodes.edu.mx/ensh/tesis/Roberto.pdf.

Rosales, C. (2013). Análisis de experiencias de innovación educativa. Enseñanza \& Teaching, 31 (2), 2013, 25-68. 

ARMONIZACIÓN ENTRE LAS COMPETENCIAS DOCENTES Y DISCENTES EN EDUCACIÓN SECUNDARIA Y SUPERIOR

Salazar, R.; Tinajero, R. y Valenzuela, B. (2013). Capítulo 15. Percepción del profesorado universitario del área de la salud sobre sus principales problemas en la práctica docente en el aula. En B. Valenzuela, F. Riberiro, M. Domínguez y M. Guillén. Modelos y Prácticas para la mejora del proceso de enseñanza aprendizaje universitario. México: Pearson.

Sarlé, P. (2010). Hacer visible la inclusión en el Nivel Inicial. Inclusión Educativa: El Desafio de enseñar y aprender diversidad. Dirección de Educación Inicial: Consejo General de Educación. Gobierno entre Ríos. Descargado en septiembre de 2012. www.entrerios. gov.ar/.../1Inclusión-Educativa-Eldesafíode enseñar-y-aprender.

Secretaría de Educación y Cultura (2015). Estadísticas del Sistema Educativo Sonora 20132014. Descargado en http://www.snie.sep.gob.mx/descargas/estadistica_e_indicadores/estadistica_e_in dicadores_educativos_26SON.pdf.

Serrano, R. y Pontes, A. (2015). El desarrollo de la identidad profesional docente en la formación inicial del profesorado de secundaria. Enseñanza \& Teaching, 34 (1), 2016, 35-55.

Schön, D. (1992). La formación de profesionales reflexivos. Madrid: Paidós-MEC.

Tonda, P. y Medina, A. (2013). La formación del profesorado en la competencia evaluadora: un camino hacia la calidad educativa. Enseñanza E Teaching, 31 (2), 2013, 167-188.

Tuning (2005). Proyecto Tuning América Latina. Descargado el 9 de noviembre de 2009. http//:www.tuning.unideusto.org/tunningal.

Valenzuela, B. A.; Huerta, T.; Rodríguez, P.; Campa, R. y Hurtado, A. (2013). Armonización de las competencias docentes y discentes de los profesores y estudiantes de educación superior y secundaria. En B. Valenzuela, F. Ribeiro, C. Domínguez y M. Guillén. Modelos y Prácticas para la mejora del proceso enseñanza aprendizaje universitario. México: Pearson. ISBN: 978-607-32-2087-3.

Vega, A. (2006). El movimiento de la discapacidad: un nuevo reto educativo. En S. Gento. Propuesta para una acción educativa de calidad en el tratamiento educativo de la diversidad. Revista Complutense de Educación, 17 (2), 13-34. ISSN: 1130-2496. Descargado en octubre de 2012. revistas.ucm.es/index.php/RCED/article/download/.../15786.

Zabalza, M. (2007). Competencias docentes del profesorado universitario. Calidad y desarrollo profesional (2. ${ }^{\mathrm{a}}$ ed., $1 .^{\mathrm{a}}$ reimp.). España: Narcea. 